\title{
II. CORES FROM BASINS ADJACENT TO THE ATLANTIC OCEAN
}

V28-122

The study of this core was undertaken to measure the benthic-planktonic age difference for the "Boyle water" of glacial time in the Caribbean Sea (see Figs 5, 6; Table 5).

\section{REFERENCES}

Boyle, E A and Keigwin, L D, 1987, North Atlantic circulation during the last 20,000 years linked to high-latitude surface temperature: Nature, v 330, p 35-40.

Broecker, W S, Andrée, M, Bonani, G, Mix, A, Klas, M, Wolfli, W and Oeschger, H, ms in preparation, Differences between the radiocarbon ages of coexisting planktonic foraminifera.

Broecker, W S, Andrée, M, Bonani, G, Wolfli, W, Oeschger, H, Klas, M, Mix, A and Curry, W, $\mathrm{ms}$ in preparation, The radiocarbon age of deep water in the glacial ocean.

Oppo, D W and Fairbanks, R, 1987, Variability in the deep and intermediate water circulation of the Atlantic Ocean during the past 25,000 years: Northern Hemisphere modulation of the southern ocean: Earth \& Planetary Sci Letters, v 86, no. 1, p 1-15. 22,000 years: Paleooceanography.

Prell, W L, 1978, Upper Quaternary sediments of the Colombia Basin: Spatial and stratigraphic variation: Geol Soc America Bull, v 89, p 1241-1255.

V28-122 CARIBBEAN SEA

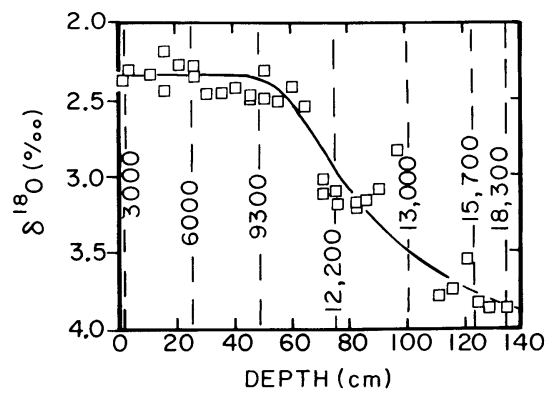

Fig 5. Oxygen isotope record for benthic foraminifera (Oppo \& Fairbanks, in press)

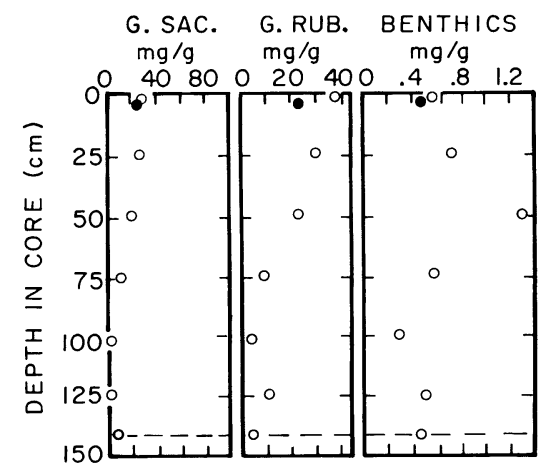

Fig 6. Abundance $v s$ depth planktonic and mixed benthic foraminifera shells for V28- 
TABLE 5

V28-122 Caribbean Sea Columbia Basin Location $\left(11^{\circ} 56^{\prime} \mathrm{N}, 78^{\circ} 41^{\prime} \mathrm{W}\right)$ Depth $3623 \mathrm{~m}$

\begin{tabular}{|c|c|c|c|c|c|c|c|c|c|c|}
\hline $\begin{array}{cc}\text { Depth } & \\
& \mathrm{fr} \\
(\mathrm{cm}) & \end{array}$ & $\begin{array}{l}\text { Coarse } \\
\text { fraction } \\
(\%)\end{array}$ & $\begin{array}{l}\text { Foram } \\
\text { sp }\end{array}$ & $\begin{array}{l}\text { Abund } \\
\text { (no./gm) }\end{array}$ & $\begin{array}{l}\text { Abund } \\
\text { (mgm/gm) }\end{array}$ & $\begin{array}{l}\text { No. tests } \\
\text { analyzed }\end{array}$ & $\begin{array}{c}\text { Weight } \\
\text { analyzed } \\
\text { (mgm) }\end{array}$ & $\begin{array}{l}\text { Date of A } \\
\text { analysis }\end{array}$ & $\begin{array}{l}\text { AMS } \\
\mathrm{s}\end{array}$ & $\begin{array}{l}\text { ge } \\
\text { (yr) }\end{array}$ & $\operatorname{Ref} *$ \\
\hline $\begin{array}{l}2-5 \mathrm{TW} \\
" " \\
"\end{array}$ & $\begin{array}{c}\text { W } 23.1 \\
" 1\end{array}$ & $\frac{\frac{G}{G}}{\frac{\text { sacc }}{\text { ruber }}} \frac{\text { benth }}{\underline{y}}$ & $\begin{array}{r}437 \\
1210 \\
19.6\end{array}$ & $\begin{array}{c}25.7 \\
23.6 \\
0.47\end{array}$ & $\begin{array}{l}241 \\
554 \\
172\end{array}$ & $\begin{array}{r}14.2 \\
10.8 \\
4.2\end{array}$ & $\begin{array}{c}\text { Mar } 87 \\
- \\
-\end{array}$ & $\begin{array}{r}3180 \\
- \\
-\end{array}$ & \pm 160 & \\
\hline $\begin{array}{l}1-3 \\
" 1 "\end{array}$ & $\begin{array}{c}30.3 \\
" ~\end{array}$ & $\frac{\frac{G}{G}}{\frac{\text { sacc }}{\text { ruber }}} \frac{\text { benth }}{\underline{\text { ben }}}$ & $\begin{array}{r}61.2 \\
2300 \\
27.2\end{array}$ & $\begin{array}{c}29.0 \\
38.7 \\
0.57\end{array}$ & $\begin{array}{l}194 \\
493 \\
530\end{array}$ & $\begin{array}{r}9.2 \\
8.3 \\
11.1\end{array}$ & $\begin{array}{c}\text { Mar } 86 \\
" ~\end{array}$ & $\begin{array}{l}2930 \\
3040 \\
3280\end{array}$ & $\begin{array}{l} \pm 120 \\
\pm 130 \\
\pm 140\end{array}$ & $\begin{array}{l}15,16 \\
15,16 \\
16\end{array}$ \\
\hline $\begin{array}{c}24-25 \\
" ~\end{array}$ & $\begin{array}{c}24.9 \\
" ~\end{array}$ & $\frac{\frac{G}{G}}{\frac{\text { sacc }}{\text { ruber }}} \frac{\underline{\text { benth }}}{\underline{n}}$ & $\begin{array}{r}595 \\
1780 \\
22.5\end{array}$ & $\begin{array}{c}27.0 \\
30.7 \\
0.73\end{array}$ & $\begin{array}{l}205 \\
610 \\
130\end{array}$ & $\begin{array}{r}9.3 \\
10.5 \\
4.2\end{array}$ & $\begin{array}{l}- \\
- \\
-\end{array}$ & $\begin{array}{l}5940 \\
6170\end{array}$ & $\begin{array}{l} \pm 130 \\
\pm 190 \\
-\end{array}$ & $\begin{array}{l}15 \\
15\end{array}$ \\
\hline $\begin{array}{c}48-49 \\
" ~\end{array}$ & $\begin{array}{c}21.4 \\
" ~\end{array}$ & $\frac{\frac{G}{G}}{\frac{\text { sacc }}{\text { ruber }}} \frac{\text { benth }}{\underline{\text { benth }}}$ & $\begin{array}{r}368 \\
1600 \\
32.2\end{array}$ & $\begin{array}{c}20.4 \\
23.4 \\
1.32\end{array}$ & $\begin{array}{l}222 \\
607 \\
207\end{array}$ & $\begin{array}{r}12.3 \\
8.9 \\
8.5\end{array}$ & $\begin{array}{l}- \\
- \\
-\end{array}$ & $\begin{array}{r}9230 \\
9390 \\
10,120\end{array}$ & $\begin{array}{l} \pm 150 \\
\pm 160 \\
\pm 200\end{array}$ & $\begin{array}{l}15,16 \\
15,16 \\
16\end{array}$ \\
\hline $\begin{array}{c}74-75 \\
"\end{array}$ & $\begin{array}{c}13.8 \\
" ~\end{array}$ & $\frac{\frac{G}{G}}{\frac{\text { sacc }}{\text { ruber }}} \frac{\text { benth }}{\underline{\text { bent }}}$ & $\begin{array}{c}177 \\
628 \\
31.2\end{array}$ & $\begin{array}{l}9.9 \\
9.4 \\
0.69\end{array}$ & $\begin{array}{l}193 \\
500 \\
367\end{array}$ & $\begin{array}{r}10.8 \\
7.5 \\
8.1\end{array}$ & $\begin{array}{l}- \\
- \\
-\end{array}$ & $\begin{array}{l}12,040 \\
12,410 \\
12,620\end{array}$ & $\begin{array}{l} \pm 220 \\
\pm 230 \\
\pm 210\end{array}$ & $\begin{array}{l}15,16 \\
15,16 \\
16\end{array}$ \\
\hline $\begin{array}{c}98-104 \\
" \\
"\end{array}$ & $\begin{array}{c}4.1 \\
" 1\end{array}$ & $\frac{\frac{G}{G}}{\frac{\text { sacc }}{\text { ruber }}} \frac{\underline{\text { benth }}}{\underline{y}}$ & $\begin{array}{c}16.6 \\
183 \\
6.50\end{array}$ & $\begin{array}{l}1.1 \\
3.3 \\
0.29\end{array}$ & $\begin{array}{l}205 \\
545 \\
227\end{array}$ & $\begin{array}{r}13.9 \\
9.8 \\
10.3\end{array}$ & $\begin{array}{c}\text { Mar } 87 \\
- \\
\text { Mar } 87\end{array}$ & $\begin{array}{l}12,650 \\
13,240 \\
15,200\end{array}$ & $\begin{array}{l} \pm 250 \\
\pm 240 \\
\pm 300\end{array}$ & $\begin{array}{l}15,16 \\
15,16 \\
16\end{array}$ \\
\hline $\begin{array}{c}123-124 \\
" \\
123-128 * *\end{array}$ & $\begin{array}{c}6.0 \\
" 1 \\
* \quad "\end{array}$ & $\frac{\frac{G}{G}}{\frac{\text { sacc }}{\text { ruber }}} \frac{\underline{\text { benth }}}{\underline{n}}$ & $\begin{array}{l}38.3 \\
549 \\
17.0\end{array}$ & $\begin{array}{c}2.2 \\
10.6 \\
0.51\end{array}$ & $\begin{array}{l}174 \\
525 \\
302\end{array}$ & $\begin{array}{r}10.1 \\
10.1 \\
8.2\end{array}$ & $\begin{array}{l}- \\
- \\
-\end{array}$ & $\begin{array}{l}15,860 \\
15,540 \\
16,550\end{array}$ & $\begin{array}{l} \pm 260 \\
\pm 270 \\
\pm 270\end{array}$ & $\begin{array}{l}15,16 \\
15,16 \\
16\end{array}$ \\
\hline $\begin{array}{c}129-139 \\
" \\
"\end{array}$ & $\begin{array}{c}8.0 \\
" \\
"\end{array}$ & $\frac{\frac{G}{G}}{\frac{\text { sacc }}{\text { ruber }}} \frac{\text { benth }}{\underline{\text { bent }}}$ & $\begin{array}{c}59.2 \\
374 \\
16.3\end{array}$ & $\begin{array}{l}4.0 \\
7.1 \\
0.46\end{array}$ & $\begin{array}{l}121 \\
582 \\
505\end{array}$ & $\begin{array}{r}8.2 \\
11.1 \\
15.3\end{array}$ & $\begin{array}{c}\text { Mar } 87 \\
- \\
\text { Mar } 87\end{array}$ & $\begin{array}{l}17,910 \\
18,730 \\
18,530\end{array}$ & $\begin{array}{l} \pm 400 \\
\pm 480 \\
\pm 420\end{array}$ & $\begin{array}{l}16 \\
16 \\
16\end{array}$ \\
\hline $\begin{array}{c}145-146 \\
"\end{array}$ & - & $\frac{G}{G} \frac{\text { sacc }}{\text { ruber }}$ & $\begin{array}{l}28.4 \\
274\end{array}$ & $\begin{array}{l}1.5 \\
4.2\end{array}$ & - & - & - & & - & \\
\hline $\begin{array}{c}157-158 \\
"\end{array}$ & - & $\underline{G} \frac{\text { sacc }}{\text { ruber }}$ & $\begin{array}{l}20.3 \\
268\end{array}$ & $\begin{array}{l}1.1 \\
4.7\end{array}$ & - & - & - & & - & \\
\hline
\end{tabular}

*Publication no. in which radiocarbon date has been published (see references cited)

$\star * 55.3 \%$ from $123-124 \mathrm{~cm}$

$26.2 \%$ from $125 \mathrm{~cm}$

$18.5 \%$ from $128 \mathrm{~cm}$ 